\title{
Solvation pressure in a Lennard-Jones fluid by molecular dynamics simulation
}

\author{
John P. Everett and David A. Faux \\ Advanced Technology Institute, University of Surrey, Guildford GU2 4ES, United Kingdom \\ (Received 16 April 2008; revised manuscript received 16 June 2008; published 15 August 2008)
}

\begin{abstract}
We present molecular dynamics simulations of a simple dimer immersed in a Lennard-Jones (LJ) fluid to test the validity of the solvation pressure model in a system where we attempt to isolate the general and ubiquitous solvation pressure. The solvated dimer acts as a simple pressure gauge in a system where the cohesive energy density (CED) of the solvent is fixed. We study the dimer bond length as a function of uniform hydrostatic pressure and as a function of a scaling parameter $x$ which changes the attractive component of the LJ solventsolute interaction. For small $x$, or weak solvent-solute attractive interactions, the liquid bond lengths are dominated by repulsive interactions, but at higher values of $x$ strong solvent-solute interactions produce a "packing effect" that is the dominant factor. We find that the change in bond length between vapor and liquid is consistent with the solvation pressure model for only a narrow range of $x$. Despite the simplicity of the system, departure from the solvation pressure model and an increase in the dimer bond length with increasing pressure are observed which is consistent with experimental observations of real liquids and normally attributed to masking effects. The existence and impact of these effects are explained in terms of CED, axial forces, and axial pressures.
\end{abstract}

DOI: 10.1103/PhysRevB.78.054113

PACS number(s): 61.20.Ne, 61.20.Ja, 31.70.Dk

\section{INTRODUCTION}

Recent years have seen increased interest in the role that solvation pressure plays in the shortening and lengthening of molecular bonds in solvated molecules. One reason for this is that many biological processes are sensitive to externally applied pressure. For example, pressure can increase the folding rates of proteins which undergo reversible folding/ unfolding transitions when subjected to hydrostatic pressures of 2-10 katm. ${ }^{1,2}$ Although the consensus is that both pressure and temperature are potential denaturants of proteins, the former has the advantage that it affects only the volume of the system whereas the latter involves changes in both the volume and thermal energy. ${ }^{3}$ Attention is focused in this area because proteins are now considered for their potential use as biosensors ${ }^{4}$ and since protein misfolding can have serious consequences including Alzheimer and Parkinson diseases. ${ }^{5}$ Thus, a clear understanding of the specific physical principles governing the solvation pressure mechanism offers a possible means for controlling pressure-dependent biological functions.

Solvent-solute interactions can modify significantly the solvent structure in the region surrounding a solute particle, the cybotactic region, from that in the pure solvent. This leads to changes in the solvation pressure experienced by solvated molecules and good evidence for the solvation pressure effect has been observed using Raman spectroscopy for nanosized objects such as carbon nanotubes, ${ }^{6,7}$ starch grains, ${ }^{8}$ pure solvents, ${ }^{9,10}$ and for solvent mixtures. ${ }^{11,12}$

The solvation pressure model asserts that all solutes, including particles immersed in their own liquid, experience a pressure equal to the cohesive energy density (CED) of the solvent, which is defined as the energy of vaporization per unit volume. This can be obtained from both experimental measurements and molecular dynamics (MD) simulations using the molar enthalpy of vaporization $\Delta H_{\text {vap }}$ and molar internal energy change on vaporization $\Delta u_{\text {vap }}$, respectively:

$$
\mathrm{CED} \equiv \frac{\Delta u_{\mathrm{vap}}}{v} \cong\left(\Delta H_{\text {vap }}-R T\right) / v,
$$

where $v$ is the molar volume of the solvent. The two molar energies are related by

$$
H_{\text {vap }}=u_{\text {vap }}+P v_{\text {vap }},
$$

where $v_{\text {vap }}$ is the molar volume of the vapor and $P$ is the pressure. A similar expression can be written for the liquid phase and since $v_{\text {vap }} \gg$, it follows that

$$
\Delta H_{\mathrm{vap}}=\Delta u_{\mathrm{vap}}+R T,
$$

assuming the van der Waals formula holds for the vapor. The CED is often expressed in units of pressure and for condensed argon, water, ethanol, chloroform, and benzene is about $1.8,{ }^{14} 24,7,3$, and $3.5 \mathrm{katm}$, respectively. ${ }^{13}$

Several theoretical models have been used to describe the solvation pressure mechanism. Schweizer and Chandler invoke competition between short-range (hard-sphere) and long-range solvent-solute interactions. ${ }^{14-16}$ Good agreement with experiment can be secured but the fitting parameters are sensitive to the hard-sphere radius of the solvent and the length scales of attractive solvent-solute interactions. ${ }^{15}$ This has focused effort on simpler models, such as the solvation pressure model, where the pressure effect of a solvent on a solute can be obtained simply from the CED of the liquid.

Dack's model correlates the internal pressure $p_{i}$ with the CED of a liquid system. ${ }^{17}$ Other workers have tested the applicability of this model to nonpolar and polar hydrogenbonded liquids and have shown that for low polar liquids the CED can be equated to internal pressure. In contrast, for strongly hydrogen-bonded liquids $p_{i}$ and CED do not reflect the same physical property. ${ }^{18}$ Moreover, liquid comparisons have shown that even when there is an equivalent internal pressure it can correspond to a different structural state and hence significantly different CED. ${ }^{16,19}$ Liquid systems whose properties are close to those of a van der Waals fluid are the 
exception because here the cohesive energy density and internal pressure are approximately equal for the liquids being compared. $^{16}$

The internal pressure and CED of a liquid, where the former is a measure of the change in internal energy of $1 \mathrm{~mol}$ of solvent as it undergoes a small isothermal expansion, are related by

$$
p_{i}=\left(\frac{\partial u}{\partial v}\right)_{T}=\frac{n \Delta u_{\mathrm{vap}}}{v},
$$

where $n$ is a dimensionless ratio which has been related to the strength of the intermolecular forces in the liquid. ${ }^{20}$ For many liquid systems, the small isothermal expansion may not disrupt all intermolecular interactions associated with the $1 \mathrm{~mol}$ of solvent and so the $p_{i}$ will diverge from the CED. For example, for highly-polar liquids such as water $n \approx 0.07$ and the CED deviates strongly from the internal pressure whereas for nonpolar liquids such as benzene $n \approx 1$ and the CED and internal pressure are approximately equal. ${ }^{21}$ Thus, the relationship between the CED and $p_{i}$ may indicate the likely validity of the solvation pressure model. So cohesion, and hence CED, results in a ubiquitous solvation pressure exerted on solutes which can be masked in the presence of strong specific interactions. For example, MD simulations of pure ethanol report that the change in bond length with pressure is well described by the solvation pressure model for all bonds except for $\mathrm{O}-\mathrm{H}$, which is lengthened with applied pressure due to masking by hydrogen bonding forces. ${ }^{9}$ In addition, a comparison between experimental data and MD simulations demonstrate that masking interactions exist in real chloroform which can be reduced or eliminated in simplified simulations. ${ }^{10}$

The different length behaviors observed have left workers cautious as to the general applicability of the model to hydrogen-bonded systems, where the vibrational properties of bonds are sensitive to local structure. It has been shown that the results of experimental studies of the change in vibrational frequencies associated with solvated molecular bonds can be interpreted in terms of a general solvation pressure added to atom-specific masking interactions. Furthermore, it has been suggested that Schweizer and Chandler's theory, adapted to include solvent-solvent effects, could achieve decomposition of the molecular interactions into solvation pressure plus masking interactions. However, the key message from previous tests of the validity of the solvation pressure model using Raman spectroscopy and MD simulations is that simplifications of the potentials appear to reduce or eliminate masking the interactions that exist in real liquids.

In this paper we examine the solvation pressure effect on a simple nonpolar dimer as vapor and immersed in a Lennard-Jones fluid. The Lennard-Jones (LJ) fluid has been the focus of a number of studies ${ }^{22,23}$ and here we use this model in an attempt to isolate the general solvation pressure by studying a liquid system where we expect $\left|p_{i}\right| \cong|\mathrm{CED}|$ such that the potentials are sufficiently simple to suggest that masking effects are reduced or eliminated. Moreover, here the solvated dimer acts merely as a pressure gauge and we use direct measurements of its bond behavior to test for the ubiquitous effects of solvation pressure and hence the validity of the solvation pressure model.

\section{MOLECULAR DYNAMICS SIMULATIONS}

Two simple systems were considered: first a 6084 particle pure LJ fluid; second the same LJ fluid but with 100 particles replaced by 100 nonpolar dimer molecules. The MD package DLPOLY 2.17 (Ref. 24) was used for all liquid systems studied.

\section{A. Simulation of pure $\mathbf{L J}$ fluid}

The pure fluid consists of $N=6084$ solvent particles interacting via the LJ (12-6) potential

$$
U_{\mathrm{SS}}(r)=4 \varepsilon_{S}\left[\left(\frac{\sigma_{S}}{r}\right)^{12}-\left(\frac{\sigma_{S}}{r}\right)^{6}\right],
$$

where $\sigma_{S}$ and $\varepsilon_{S}$ are the usual LJ length and energy parameters, respectively and the subscript identifies the solvent. The solvent parameters are typical for benzene, $\sigma_{S}=5 \AA$ and $\varepsilon_{S} / k_{B}=414 \mathrm{~K},{ }^{25,26}$ which is a nonpolar solvent with a CED of around $3.5 \mathrm{katm}$ and a fairly wide liquid range of around $75 \mathrm{~K}(278.7-353.3 \mathrm{~K}){ }^{19,25}$

The pure liquid consists of a cube containing the solvent particles initially arranged in a face-centered cubic (fcc) lattice, in a volume $V$, at a density of $800 \mathrm{~kg} \mathrm{~m}^{-3}$, which is approximately $10 \%$ lower than the experimental density of liquid benzene. All simulations of the pure liquid and liquiddimer mixture were performed at $293 \mathrm{~K}$. Periodic boundary conditions were employed in the normal way and the LJ potentials were truncated at a cutoff $r_{\text {cut }}=2.5 \sigma_{S}$ with the usual LJ tail corrections applied. ${ }^{27}$ Equilibration of the pure liquid was achieved by allowing repeated isotropic contractions for $10^{6}$ time steps of 0.2 fs in the $N-P-T$ ensemble (constant number of particles, pressure, and temperature) with a zero pressure target. The system was then thermalized further in the $N-V-E$ ensemble (constant number of particles, volume, and energy) using the cell dimensions obtained from the $N-P-T$ thermalization and equilibration was verified by ensuring that there was no systematic drift in pressure. This procedure produced a stable equilibrated liquid system at zero pressure with extremely small pressure fluctuations of \pm 1 atm

There are two methods for calculating the CED of the liquid from the simulation. The most straightforward approach is to evaluate

$$
\mathrm{CED}_{S}=\frac{-u_{S}}{v_{S}},
$$

where $u_{S}$ and $v_{S}$ are the mean configurational energy and volume per solvent particle, respectively. An alternative approach for the calculation of the CED examines the interactions between a particle and those particles in its immediate surroundings. The effective CED due to the surrounding particles can be obtained by considering the breaking and formation of bonds when a particle is inserted into the liquid. Suppose each particle, on average, forms bonds to $c$ neighboring particles, where $c$ can be considered the coordination 
number. In the LJ liquid we would expect $c \approx 12$ for first nearest neighbors as for a fcc lattice. ${ }^{34}$ A cavity is formed by breaking $c / 2$ bonds and then a particle is inserted into the cavity forming $c$ bonds. The net result is the formation of $c / 2$ bonds.

In a liquid of course, $c$ is a function of $r$ and is related to the solvent-solvent radial distribution function $(\mathrm{rdf}) g_{\mathrm{SS}}(r)$,

$$
c(r)=4 \pi \rho_{N} \int_{0}^{r} r^{2} g_{\mathrm{SS}}(r) d r
$$

where $\rho_{N}$ is the average number density $N / V=1 / \nu_{S}$. It is then evident that the configurational energy is given by

$$
u_{S}=2 \pi \rho_{N} \int_{0}^{\infty} U_{\mathrm{SS}}(r) g_{\mathrm{SS}}(r) r^{2} d r
$$

where $U_{\mathrm{SS}}(r)$ is the solvent-solvent LJ pair potential given by Eq. (5) and the CED is obtained using Eq. (6). For a singlespecies liquid, the CED obtained using either Eq. (5) for the system as a whole or using Eq. (8) for the local CED should produce the same result. Equation (8) can be adapted, however, to estimate the effective local CED experienced by the dimer solute molecules as explained in the following section. ${ }^{28}$

\section{B. Simulation of solvent-dimer mixture}

This system consists of a binary mixture of $N_{D}=100$ nonpolar dimer solutes immersed in a $N_{S}=5984$ particle LJ solvent. The intramolecular dimer bond is described by a Morse potential

$$
U(r)=E_{0}\left(\left\{1-\exp \left[-k\left(r-r_{0}\right)\right]\right\}^{2}-1\right),
$$

with parameters that are typical of those used to model the single $\mathrm{O}-\mathrm{O}$ bond in hydrogen peroxide which is liquid at

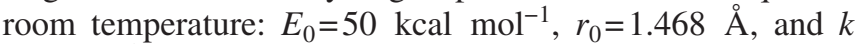
$=1.375 \AA^{-1} \cdot 29,30$

The solvent-solvent interaction pair potential and parameters in the mixture are the same as those of the pure fluid presented in the previous section. For solvent-solute interactions, the dimer is treated as two-site and the interaction between a solvent particle and each dimer site is modeled as LJ (12-6) but modified by a parameter $x$ that scales the attractive term:

$$
U_{\mathrm{SD}}(r)=4 \varepsilon_{X}\left[\left(\frac{\sigma_{X}}{r}\right)^{12}-x\left(\frac{\sigma_{X}}{r}\right)^{6}\right],
$$

where $\sigma_{X}$ and $\varepsilon_{X}$ are the LJ length and energy parameters, respectively, for the solute-solvent interactions and are constants independent of $x$. This expression may be rewritten in the form

$$
U_{\mathrm{SD}}(r)=4 \varepsilon_{\mathrm{SD}}(x)\left[\left(\frac{\sigma_{\mathrm{SD}}(x)}{r}\right)^{12}-\left(\frac{\sigma_{\mathrm{SD}}(x)}{r}\right)^{6}\right],
$$

such that

$$
\sigma_{\mathrm{SD}}(x)=\sigma_{X} x^{-1 / 6}
$$

TABLE I. The solvent-solute LJ energy and length parameters. In all simulations the solvent length and energy parameters are $\sigma_{S}=5 \AA$ and $\varepsilon_{S} / k_{B}=414 \mathrm{~K}$, respectively.

\begin{tabular}{lcl}
\hline \hline$x$ & $\begin{array}{c}\varepsilon_{\mathrm{SD}} / k_{B} \\
(\mathrm{~K})\end{array}$ & $\begin{array}{l}\sigma_{\mathrm{SD}} \\
(\AA)\end{array}$ \\
\hline 0.5 & 39.9 & 4.46 \\
1 & 159.7 & 3.98 \\
2.1 & 704.3 & 3.50 \\
3 & 1437 & 3.31 \\
4 & 2555 & 3.16 \\
\hline \hline
\end{tabular}

$$
\varepsilon_{\mathrm{SD}}(x)=x^{2} \varepsilon_{X},
$$

thus satisfying the requirement that the repulsive component of the solvent-solute potential is a constant. Since the solvent-solvent length and energy parameters are fixed, any variation in $x$ represents a variation in the energy and length parameters associated with the solute only. For the solventsolute parameters we expect the Lorentz-Berthelot mixing rules to hold, thus ${ }^{30}$

$$
\begin{gathered}
\sigma_{\mathrm{SD}}(x)=\frac{1}{2}\left[\sigma_{S}+\sigma_{D}(x)\right], \\
\varepsilon_{\mathrm{SD}}(x)=\left[\varepsilon_{S} \varepsilon_{D}(x)\right]^{1 / 2},
\end{gathered}
$$

where each dimer site, or atom, has energy and length parameters $\varepsilon_{D}(x)$ and $\sigma_{D}(x)$, respectively, assigned oxygenoxygen $\mathrm{LJ}$ interaction parameters for the case $x=1$. Hence $\sigma_{D}(x=1)=2.95 \AA$ and $\varepsilon_{D}(x=1) / k_{B}=61.6 \mathrm{~K} .{ }^{31}$ Also, in Eq. (10), $\varepsilon_{X}=\varepsilon_{\mathrm{SD}}(x=1) / k_{B}=159.7 \mathrm{~K}$ and $\sigma_{\mathrm{SD}}(x=1)=3.98 \AA$. These choices lead to the solvent-solute LJ parameters listed in Table I. Note that there are no solute-solute intermolecular interactions.

For simulations of the mixture, 6084 solvent particles were arranged in a fcc lattice and 100 were replaced with dimers. Starting with $x=1$, equilibration began with repeat $N-P-T-N-V-T$ cycles for $10^{6}$ time steps of 0.2 fs which produced simulation cells containing the mixture at a range of pressure from about 0 to $1.2 \mathrm{katm}$. In order to obtain systems with different values of $x$ at approximately the same volume and hence density, the equilibrated system with $x=1$ was equilibrated for a new $x$ in the $N-V$-T ensemble for $5 \times 10^{6}$ time steps of $0.2 \mathrm{fs}$ and then in the $N-V-E$ ensemble for a maximum of $3 \times 10^{6}$ time steps of $0.2 \mathrm{fs}$. As before, equilibration was verified and the simulation terminated when there was no pressure or bond length drift.

Each cell was then simulated using the $N-V-E$ ensemble for a maximum of $2 \times 10^{6}$ time steps of $0.2 \mathrm{fs}$ with the thermodynamic data and atomic positions recorded every 25 time steps. Dimer bond length measurements were obtained by averaging the individual bond length measurements of the 100 dimers over all recorded time steps. The pressure and bond length data were monitored throughout each run and for the mixture equilibration was verified and the simulation terminated when there was no systematic drift in pressure or bond length. Each cell was then taken through a cycle of four 
simulations using the $N-V-E$ ensemble for $5 \times 10^{6}$ time steps of $0.2 \mathrm{fs}$, again with the thermodynamic data and atomic positions recorded every 25 time steps, and bond lengths were obtained at a given pressure by averaging the bond lengths obtained from each of the four $N-V-E$ runs. The standard deviation of the pressure and temperature fluctuations was typically $0.015 \mathrm{katm}$ and $1.5 \mathrm{~K}$, respectively. It is also noted that the use of the $N-V-E$ ensemble at the data taking stage is essential as the normal thermostat (Nosé-Hoover) for $N-V-T$ ensembles scales the atomic velocities and the normal barostat (Berendsen) for $N-P-T$ ensembles scales the volume and hence bond lengths. Both ensembles therefore interfere with the quantities we wish to measure.

By definition, a dimer as vapor will have a CED of zero and does not experience a solvation pressure. Thus, we can test for the validity of the solvation pressure model by comparing bond lengths obtained from liquid and vapor simulations. We modeled the vapor by placing a single dimer in a cubic simulation cell with the same dimensions as the liquid, which meant that the cell was sufficiently large to render negligible any interactions between the molecule and its images due to periodic boundary conditions. To obtain the vapor bond length, we averaged the bond length obtained from $10^{3}$ simulations undertaken, at zero pressure and $293 \mathrm{~K}$, using the $N-V-E$ ensemble for $10^{7}$ time steps of $0.02 \mathrm{fs}$.

The CED experienced by a dimer due to its local environment can be obtained by considering the bonds broken in the formation of a cavity and the bonds formed when a dimer is inserted in the manner described in Sec. II B. Here, however, $c / 2$ solvent-solvent bonds are broken in forming the cavity and $2 c$ solute-solvent bonds are formed when the dimer is inserted. Therefore,

$$
u_{D}=2 \pi \rho_{N} \int_{0}^{\infty} U_{\mathrm{SS}}(r) g_{\mathrm{SS}}(r) r^{2} d r-8 \pi \rho_{N} \int_{0}^{\infty} U_{\mathrm{SD}}(r) g_{\mathrm{SD}}(r) r^{2} d r
$$

and

$$
\mathrm{CED}_{X}=\frac{-u_{D}}{v_{D}}
$$

where $g_{\mathrm{SD}}(r)$ is the solvent-solute radial distribution function and $v_{D}$ is the volume per dimer. It is assumed that the particle density is approximately constant and equal to the bulk density in the region of the integrations which, in practice, is taken to $r_{\text {cut }}$. This integral cutoff incorporates the second shell in the energy calculations and consequently reduces the error since the interaction energy due to all neighbors beyond the second shell contributes only about $1.8 \%$ of the total.

\section{RESULTS AND DISCUSSION}

The zero pressure density of the pure fluid determined from the simulation was $871.7 \mathrm{~kg} \mathrm{~m}^{-3}$. Using Eq. (6), we calculated a solvent cohesive energy density of $2.3 \pm 0.3 \mathrm{katm}$. This is in excellent agreement with the CED of $2.2 \mathrm{katm}$ obtained using Eq. (8) for both the pure fluid and fluid component of the mixture. For the pure fluid we calcu-

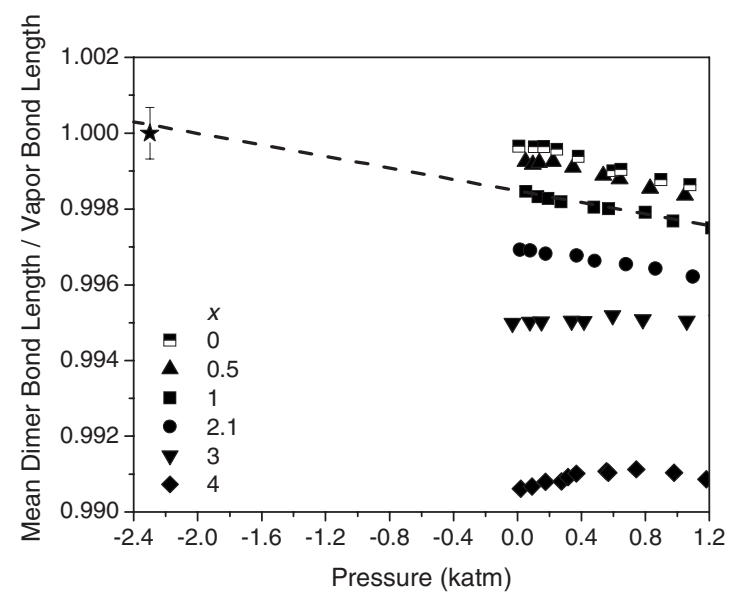

FIG. 1. The dimer bond lengths determined from MD simulations as a function of uniform hydrostatic pressure.

lated the volume per solvent particle from $V / N$, while for the mixture we calculated this quantity using

$$
v_{S}=\frac{V}{N_{S}+X_{S} N_{D}}
$$

where $N_{S}$ and $N_{D}$ are the number of solvent and solute particles in the system, respectively, and $X_{S}$ is the mole fraction of the solvent equal to 0.968 .

Figure 1 shows the average normalized dimer bond length as a function of applied pressure for various values of $x$. Also shown is the vapor bond length and since this bond will not experience the solvation pressure felt by the liquid, we plot it at $-2.3 \mathrm{katm}$, equal to the CED of the solvent. All dimer bond lengths were normalized to the vapor bond length which was equal to $1.477 \AA$. Typically, the standard deviation of the bond length fluctuations was $5 \times 10^{-4} \AA$ for the liquid and $10^{-3} \AA$ for the vapor. The applied pressure is confined to a maximum of about 1.2 katm to avoid the liquid-solid transition which occurs at higher densities.

As expected, Fig. 1 shows that the mean dimer bond length decreases as the attractive component of the solventsolute potential, characterized by $x$, is increased. The dashed line, obtained from the linear regression of the liquid data only for the case where $x=1$, passes through the vapor data within the error and it appears that it is only for this value of $x$, or narrow range of values either side of $x=1$, that the behavior of the bond length is consistent with the solvation pressure model. In other words, the application of a uniform hydrostatic pressure compresses the dimer bond length in the same manner as the solvation pressure acting on the liquid at normal pressure compared to the vapor.

For $x=0,0.5,1$, and 2.1 there is a linear compression of the bond with increasing hydrostatic pressure and all plots exhibit a similar slope. However, this is not so when $x=3$ and 4 where we observe an increase in bond length as the applied pressure is increased. The simple LJ system reveals many of the complex features observed in experiments where bond length changes are inferred from the shift of Raman peaks with applied uniform pressure. For example, in real systems where strong attractive forces such as hydrogen 

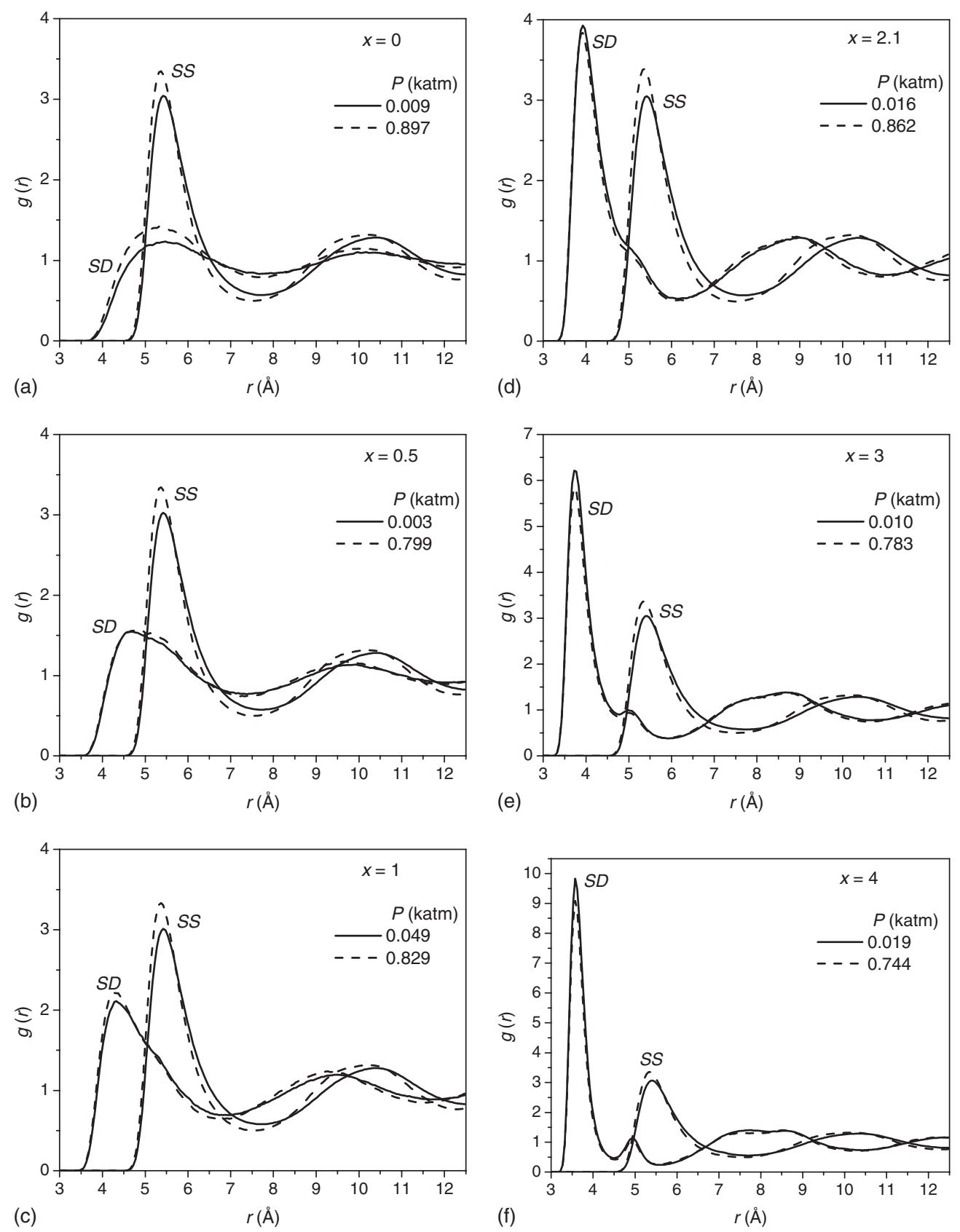

FIG. 2. The solvent-solute rdfs as a function of radial distance $r$ for (a) $x=0$, (b) $x=0.5$, (c) $x=1$, (d) $x=2.1$, (e) $x=3$, and (f) $x=4 ; D$ refers to a single dimer site. For clarity, we plot the curves at two pressures only and also include solvent-solvent rdfs.

bonding are known to be present, comparisons between Raman spectra from experiment and simulation indicate bond compression and an increase in bond length as a function of pressure. ${ }^{8-10}$ We now seek to establish why agreement with the solvation pressure model occurs for a small range of $x$ and why, for large relative attractive potentials, an increase in bond length is observed with increasing applied pressure.

It is evident that the dimers experience a local CED different from the CED of the solvent that surrounds it which, presumably, is due to the local arrangement of solvent atoms in the cybotactic region of the dimer. The expressions for the local CED given by Eqs. (16) and (17) include contributions from the rdf and dimer volume.

Figure 2 shows the solvent-solvent (SS) and solvent- solute (SD) rdfs as a function of $x$. As $x$ is increased the first peak in the solvent-solute rdfs becomes taller but narrower, which suggests a reduction in the first coordination number accompanied by tighter packing in the cybotactic region. A low and narrow second peak close to the first develops which becomes more prominent for increasing $x$. This may be thought of as a splitting of the first peak as first neighbors are squeezed out of the first shell. An increase in $x$ corresponds to a decrease in the solvent-solute LJ length parameter $\sigma_{\mathrm{SD}}(x)$ which in turn leads to a shortening of the position of the first peak as the solvent molecules are allowed to close in on dimer sites and hence the squeeze on first shell particles.

Equation (7) was used to calculate the first coordination number $c_{1}$ as a function of pressure by integrating to the first 


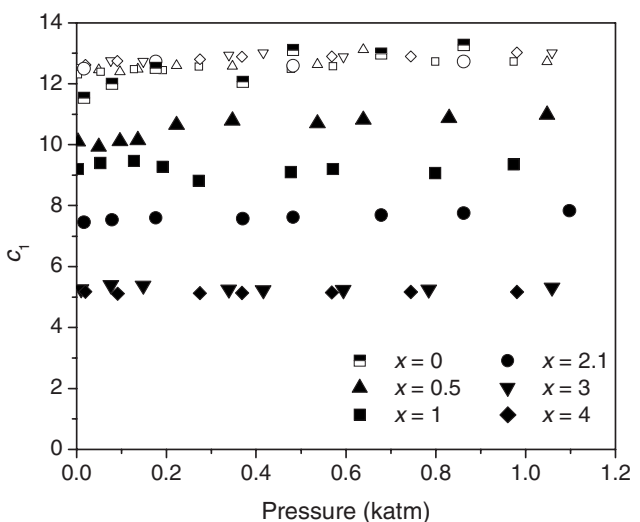

FIG. 3. The solvent-solute first coordination number $c_{1}$ for varying $x$ plotted as a function of uniform hydrostatic pressure (closed symbols). Also shown for comparison are the solvent-solvent first coordination numbers (open symbols) for equivalent values of $x$.

minimum of each rdf. $c_{1}$ is equal to the number of solvent particles within the first coordination shell of a dimer site. These data are shown in Fig. 3 as a function of applied pressure for various values of $x$ and includes the $c_{1}$ obtained from the solvent-solvent rdfs. The latter confirms that the first coordination number is about 12 , consistent with a fcc structure due to a compact liquid. For the solvent-solute coordination number at $x=0$, we find that $c_{1} \sim 12$, and from there we observe a decrease of $c_{1}$ for increasing $x$, consistent with the splitting of the first peak in the rdfs. As $x$ is increased, first shell particles are squeezed and some are forced out leaving a highly compact cybotactic region. For small $x$, the solvent-solute first coordination number increases slightly with pressure consistent with an uniform compression of the system. However, for $x=3$ and $x=4, c_{1}$ is constant and independent of pressure thus suggesting a lower limit to $c_{1}$ of about 5 .

Recall that the bond length data presented in Fig. 1 show that there are masking effects that alter the net solvation pressure experienced by the solute bond, which results in bond behavior different from that expected for a fit to the solvation pressure model. We have also demonstrated using rdfs and first coordination numbers that increasing the attractive component of the solvent-solute potential modifies significantly the solvent structure within the cybotactic region. To demonstrate further the extent of structural reorganization we adopt a method similar to that used by Laaksonen et al $^{32}$ to obtain the structural density function, or local structure, surrounding a molecule. We first translate each dimer so that its center sits at the origin and then apply rotational matrices to align the bond length along the $x$ axis. An equivalent translation and rotation is then applied to solvent particles originally falling within a radial distance of $r \leq r_{\text {cut }}$ from the center of the dimer. Over the course of a simulation we record solvent positions relative to the origin or center of the dimer bond, and obtain two-dimensional $x-y$ and $y-z$ slices of the solvent structure surrounding the dimer by restricting the recording of solvent positions where $-0.05 \AA<z<0.05 \AA$ and $-0.05 \AA<x<0.05 \AA$, respectively. Figures 4 and 5 show $x-y$ and $y-z$ slices for $x=0.5,1$, and 3 , up to a radial distance of $12 \AA$. Note that the slices show all solvent posi-

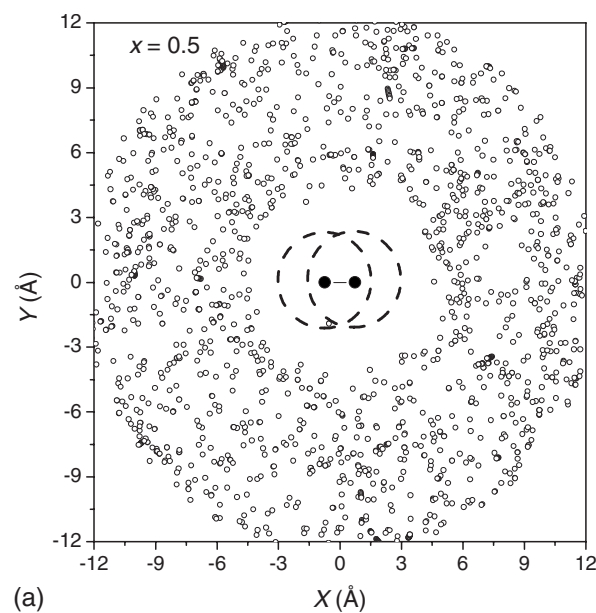

(a)

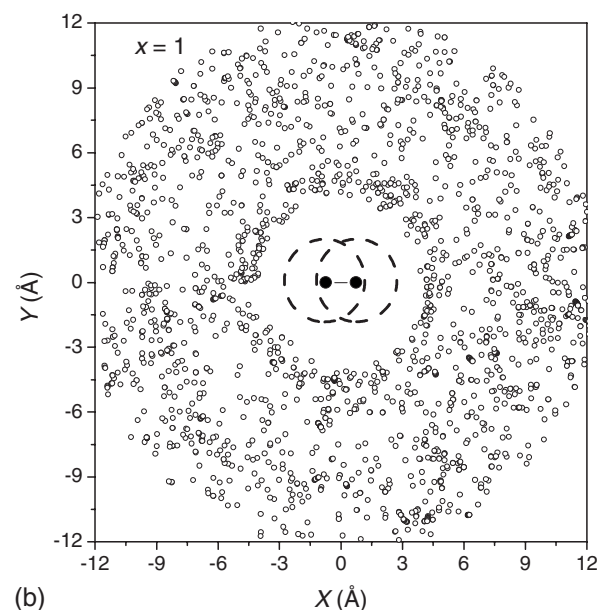

(b)

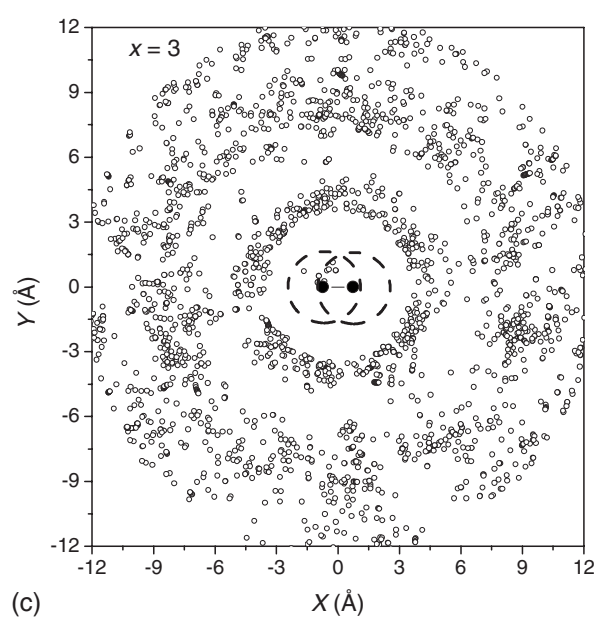

FIG. 4. Two-dimensional $x-y$ slices showing the local solvent structure with the dimer centered at the origin for (a) $x=0.5$, (b) $x=1$, and (c) $x=3$. For each $x$, the black dot and hashed curve shows the position of the center of the dimer site and the LJ circle with diameter $\sigma_{\mathrm{SD}}(x)$, respectively.

tions with the cutoff radius of the 100 dimers over 1200 mixture configurations.

The $x-y$ and $y-z$ slices obtained when $x=0.5$ manifest the wide and relatively low first peak in the solvent-solute rdf. When $x$ is increased, the position of first coordination shell becomes more prominent and appears highly localized at 


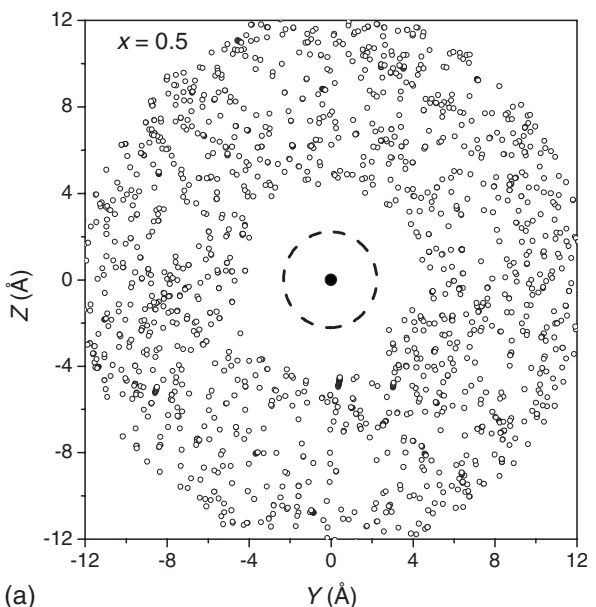

(a)

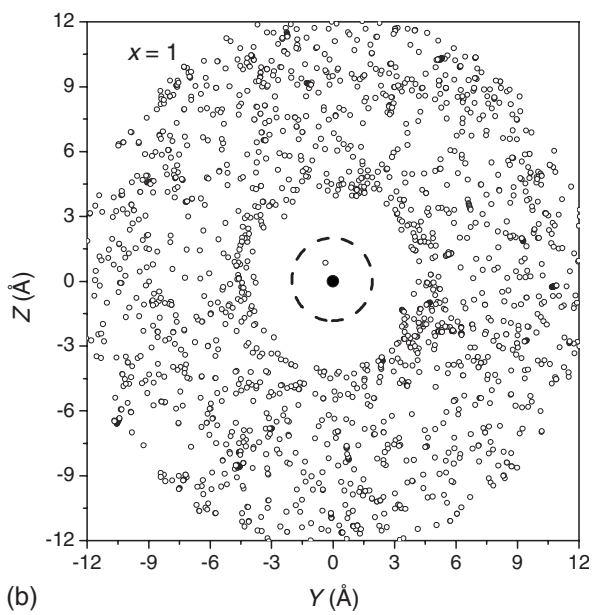

(b)

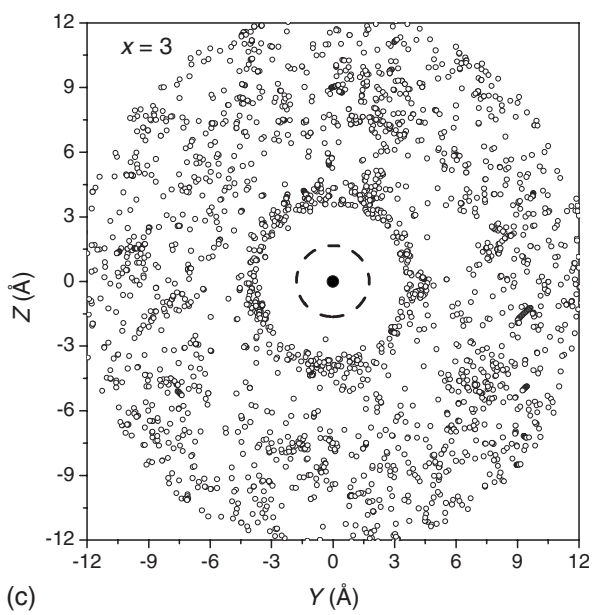

FIG. 5. Two-dimensional $y$-z slices showing the local solvent structure with the dimer centered at the origin for (a) $x=0.5$, (b) $x=1$, and (c) $x=3$. For each $x$, the black dots indicate the position of each dimer site center and the hashed curves show the LJ circles with diameter $\sigma_{\mathrm{SD}}(x)$, respectively.

$x=3$, corresponding the splitting of the first rdf peak and consequent appearance of a small second peak slightly further out. Figures 4 and 5 confirm that there is significant structural reorganization of solvent molecules surrounding the solute for large $x$.

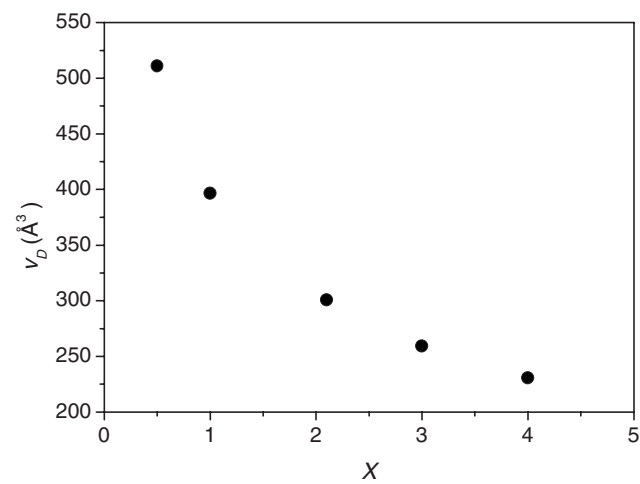

FIG. 6. The dimer volume calculated using Eq. (19) as function of the scaling parameter $x$ at zero applied pressure.

We now turn our attention to the dimer volume. As $x$ is increased, $\sigma_{\mathrm{SD}}(x)$ decreases and solvent particles move closer to the solute sites. Thus, the local packing environment of the dimer is altered and the volume occupied by the dimer is reduced leading to an increase in the local solvation pressure according to Eq. (17).

Calculating free volumes in liquids has proved a difficult task partly due to the computational and experimental difficulties associated with measuring and characterizing them. Although Krekellberg et al. ${ }^{33}$ have developed a model that predicts how the attractive strength affects the free volume distributions of a square-well fluid with short range interactions, here an elementary model is used which assumes that the dimer occupies a prolate ellipsoid with major and minor axes as illustrated in the $x-y$ and $y-z$ slices, respectively.

If the minor and major axes are $R_{1}$ and $R_{2}$, respectively, then

$$
v_{D}=\frac{4 \pi}{3}\left(R_{1}^{2} R_{2}\right)
$$

where $R_{1}$ is the distance from the dimer axis to the first peak of the rdf and $R_{2}=R_{1}+d / 2$ where $d$ is the dimer bond length.

Figure 6 confirms that the dimer volume $v_{D}$ decreases for increasing $x$ at zero applied pressure. Figure 7 shows that the local CED experienced by the dimer $\mathrm{CED}_{X}$, calculated from Eq. (17), increases as a function of $x$ consistent with the

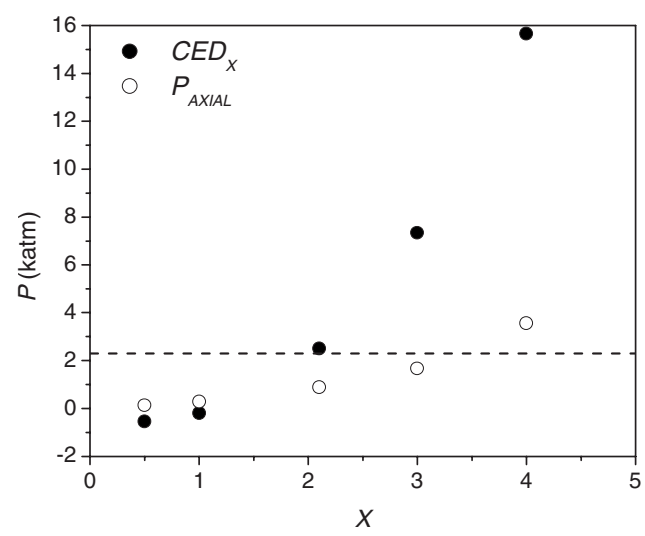

FIG. 7. A comparison of $\mathrm{CED}_{X}$ and the axial pressure $P_{\mathrm{AXIAL}}$, calculated from Eq. (20), as a function of $x$. 
changes in bond lengths presented in Fig. 1. For small $x$, $\mathrm{CED}_{X}$ is negative; for example, at $x=1, \mathrm{CED}_{X} \sim-0.2 \mathrm{katm}$. Yet, at $x=1$, the behavior of the dimer bond length is consistent with the solvation pressure model and compliance with the model implies that at zero applied pressure the bond should experience a CED equivalent to that of the solvent $C E D$, namely, about $2.3 \mathrm{katm}$. Broad agreement with the SPM, within bond length error, was obtained for approximately $0.7<x<1.6$, which corresponds to a range of solvation pressure $-0.3<\mathrm{CED}_{X}<1.4(\mathrm{katm})$ in Fig. 7, well below the solvent $\mathrm{CED}$. Note that the calculation of $\mathrm{CED}_{X}$ includes the effect of both the reduced dimer volume with increasing $x$ and also changes to the local environment characterized by the rdf. The negative CED obtained in the low $x$ regime is because the solvent-solute interactions are dominated by the repulsive component and so the affinity of solvent with solute is lower than the solvent-solute interactions. We note that other workers have used dilution to generate negative solvation pressures in liquids ${ }^{8}$ and state that these arise because pressure and dilution change the environment around solute molecules. ${ }^{34}$

At low $x, \mathrm{CED}_{X}$ is dominated by the energy of cavity creation, or break up of solvent-solvent bonds, while for higher $x$ it is the solute solvation that dominates due to the increasing strength of the attractive component of the solvent-solute potential. For $x>1$, there is both a positive CED and the increasing strength of the solvent-solute interaction leads to significant structural reorganization of solvent particles, a lower free volume, and hence a packing effect that dominates the bond behavior. At $x \sim 2, \mathrm{CED}_{X}$ corresponds with the CED of the solvent. The fact that $\mathrm{CED}_{X}$ increases with increasing $x$ suggests that the CED is a real pressure that plays a role in the observed bond compression. However, what is striking is that in our simplified system there is evidence of masking interactions that cause the bond length to deviate significantly from the simple solvent solvation pressure dependence. It would therefore be useful to compare $\mathrm{CED}_{X}$ with a direct calculation of the pressure exerted on the bond determined from the net axial force.

The net axial force $f_{\text {AXIAL }}$ is obtained by summing the bond-lengthening and bond-shortening force components due to solvent molecules lying within a distance $r \leq r_{\text {cut }}$ of the dimer where a positive or negative net force corresponds to a net shortening or lengthening force, respectively. In order to calculate a net axial pressure, it is reasonable to assume that $f_{\text {AXIAL }}$ acts on a net area of $\pi\left[\sigma_{\text {SD }}(x) / 2\right]^{2}$. Thus the net axial pressure at a given $x$ is

$$
P_{\mathrm{AXIAL}}=\frac{f_{\mathrm{AXIAL}}}{\pi\left[\sigma_{\mathrm{SD}}(x) / 2\right]^{2}}
$$

and these values are included on Fig. 7. A quadratic fit to $P_{\text {AXIAL }}$ provides a good fit and indicates that the zero pressure net axial force, and hence bond length, may be dominated by the energy parameter since $\varepsilon_{\mathrm{SD}} \infty x^{2}$.

Figure 7 suggests that the pressure mechanism controlling the bond behavior may be attributed to the internal pressure rather than the CED or solvation pressure. Evidence comes from Eq. (4) which showed that in liquids where masking interaction dominate $p_{i}<\mathrm{CED}$ which is similar to the behav-

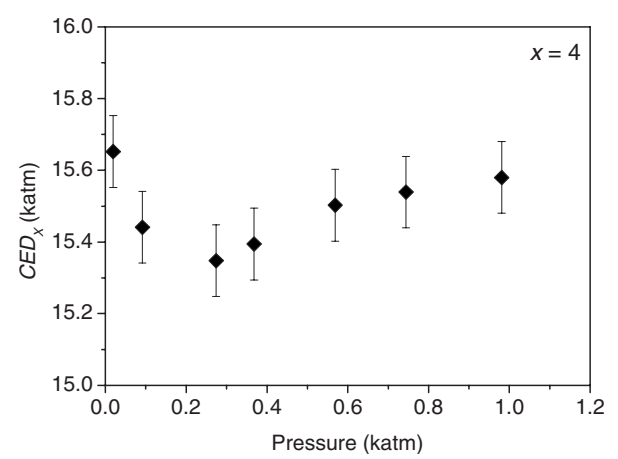

FIG. 8. The solvation pressure experienced by a dimer, calculated using Eqs. (16) and (17), shown as function of uniform hydrostatic pressure for $x=4$.

ior seen here where $P_{\text {AXIAL }}<\mathrm{CED}_{X}$. We note that the dimer bond behavior appears to fit the solvation pressure model at $x \sim 1$ which is where, as shown in Fig. $7, \mathrm{CED}_{X} \sim P_{\mathrm{AXIAL}}$.

Finally, we explain the pressure dependence of the bond lengths for large $x$ shown in Fig. 1. For $x<2.1$ the bond lengths show an approximate linear decrease for increasing applied pressure while for $x=3$ and 4 the bond lengths are lengthened with applied pressure, at least for small applied pressures.

Figures 8-10 present the $\mathrm{CED}_{X}$, dimer volume, and $P_{\text {AXIAL }}$, respectively, as functions of uniform hydrostatic pressure for $x=4$. The applied pressure promotes changes in the solvent structure that lead to a systematic increase in free volume but the change in volume is too small to significantly affect the value of $\mathrm{CED}_{X}$. The $\mathrm{CED}_{X}$ shows a small drop in value with increasing applied pressure up to a pressure of about $0.2 \mathrm{katm}$ before increasing once more. However, it is $P_{\text {AXIAL }}$ which shows a significant fall with increasing applied pressure consistent with the increase in bond length observed in Fig. 1. The lengthening of the bond is similar to the bond behavior observed in liquids where masking interactions are evident due to the presence of hydrogen bonds. The theoretical calculation of the axial pressure would be complex because it depends on the specific positions of the solvent particles in the cybotactic region. The attractive and repulsive contributions to the interatomic potentials can serve to stretch or compress the dimer bond depending on their posi-

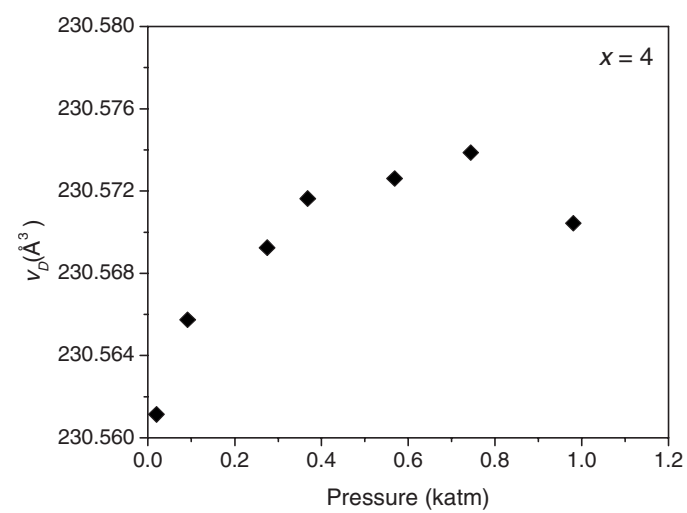

FIG. 9. The dimer free volume, calculated using Eq. (19), shown as function of uniform hydrostatic pressure for $x=4$. 


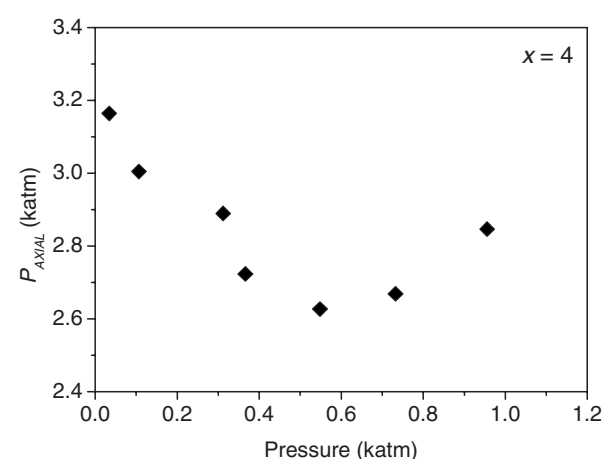

FIG. 10. The axial pressure calculated from the axial force as calculated using Eq. (20), experienced by the dimer plotted as a function of uniform hydrostatic pressure for $x=4$.

tion relative to the bond axis. The calculation of $\mathrm{CED}_{X}$ involves averaging over the rdf. It is a mean field calculation which therefore loses detail of the specific directions of the surrounding particles. The details of the bond behavior can only be fully explained if this directional information is available. Solutes which, experimentally, exhibit an increase in bond length as a function of applied hydrostatic pressure are indicative of strong, highly-directional interactions, such as hydrogen bonding. ${ }^{9}$

\section{CONCLUSION}

In this paper, we examine the effect of solvation pressure on a simple nonpolar dimer immersed in a Lennard-Jones fluid via molecular dynamics simulation. The solvated dimer acts as a pressure gauge in a system where the solvation pressure of the solvent is constant and we have attempted to isolate the general solvation pressure by simplification of the potentials. The dimer bond length is measured as a function of $x$, which scales the attractive component of the solventsolute interaction potential, and as a function of applied uniform hydrostatic pressure. While ostensibly a simple system, our results exhibited rich behavior similar to that observed experimentally, using Raman spectroscopy, for a range of solute-solvent systems including those where hydrogen bonding is known to be a dominant factor in bond length behavior. ${ }^{8-10}$ We found that for $x \sim 1$, the observed bond behavior is consistent with the solvation pressure model but with the caveat that neither the effective local CED nor the axial pressure indicate that the pressure experienced by the dimer was that expected for compliance with the model.

For weak attractive solvent-solute interactions $x<1$ we find that the dimer bond is compressed with applied pressure as the mean positions of the surrounding solvent particles draw closer to the dimer. We calculate that the dimer experiences a negative local CED but a positive net axial force and axial pressure at $x=1$. We conclude that in the low $x$ regime the bond behavior is dominated by repulsive solventsolute interactions. For $x>1$, attractive solvent-solute interactions dominate and this leads to significant structural reorganization of solvent particles surrounding the dimer solutes in which the number of first-nearest neighbors is reduced and a second peak in the rdf appears. At $x=3$ and $x=4$, the dimer bond is found to lengthen with applied pressure. This is due to the subtle structural changes where first-nearest neighbors are squeezed out leading to a reduction in the axial pressure. Information about the specific positions of the local solvent particles is required to correctly predict this behavior and this effect is only partially revealed by the CED which averages over the local particle density. The high $x$ regime corresponds to a positive CED, axial force, and axial pressure and we conclude that here the bond length behavior is dominated by attractive solvent-solute interactions and the consequent packing restrictions or so called "packing effect." An increase in dimer bond length with applied pressure is indicative of strong, attractive forces and local structure and is consistent with experimental observations in hydrogenbonded systems. ${ }^{9}$

Although the main aim of this study is to test the validity of the solvation pressure model, and hence ubiquitous nature of solvation pressure, we emphasize that all the conclusions drawn have been tested on the LJ system only. Other work worth noting is a study of how the attractive potential influences the structuring of spheres which demonstrated that cohesion between atoms is enormously important to structure and is of greater significance than first realized. ${ }^{35}$ Also, Kodaka $^{36,37}$ has reported that there is a correlation between the diameter of solvents and packing density and that the former tends to increase depending on the magnitude of CED at constant temperature and pressure. Furthermore, that hydrophobicity is caused by the packing density of water (or organic solvents such as ethylene glycol) being higher than that expected for a solvent with the same size and lower CED. Therefore, it will be interesting to see whether the "packing effect" that we have observed at high $x$ has the pronounced effect on bond behavior in real systems that we have shown to be the case for the LJ system.

In conclusion, results suggest that the bond behavior should be attributed to the net axial pressure, or internal pressure, rather than the cohesive energy density. We also found that the dimer bond behavior appears to give a fit to the solvation pressure model at $x \sim 1$, which is the value of $x$ for which $\mathrm{CED}_{X} \sim P_{\mathrm{AXIAL}}$. The solvation pressure model does not, in general, correctly describe the change in bond length from vapor to liquid except for a specific and limited ranges of $x$. Our results do not support the idea that our simple pressure gauge, the dimer, experiences a ubiquitous applied pressure equivalent to the CED of the solvent. That is, the solvation pressure model has limited validity in our simplified system where we have attempted to isolate the general solvation pressure by using simple LJ potentials.

These results should aid the interpretation of experiments in which the bond lengths within solute molecules are inferred from changes in Raman mode frequencies as a function of applied pressure and hence provide an insight into the nature of the specific solute-solvent interactions in real systems.

\section{ACKNOWLEDGMENT}

J. P. Everett acknowledges funding by the University of Surrey and Daphne Jackson Trust. 
${ }^{1}$ G. J. Vidugiris, J. L. Markley, and C. A. Royer, Biochemistry 34, 4909 (1995).

${ }^{2}$ N. H. Hillson, J. N. Onuchic, and A. E. Garcia, Proc. Natl. Acad. Sci. U.S.A. 96, 14848 (1999).

${ }^{3}$ A. Marabotti, A. Ausili, M. Staiano, A. Scirè, F. Tanfani, A. Parracino, A. Varriale, M. Rossi, and S. D' Auria, Biochemistry 45, 11885 (2006).

${ }^{4}$ M. Staiano, P. Bazzicalupo, M. Rossi, and S. D' Auria, Mol. BioSystem 1, 354 (2005).

${ }^{5}$ D. J. Selkoe, Nat. Cell Biol. 6, 1054 (2004).

${ }^{6}$ J. R. Wood, M. D. Frogley, A. D. Prins, D. J. Dunstan, and H. D. Wagner, High Press. Res. 18, 153 (2000).

${ }^{7}$ J. R. Wood, Qing Zhao, M. D. Frogley, E. R. Meurs, A. D. Prins, T. Peijs, D. J. Dunstan, and H. D. Wagner, Phys. Rev. B 62, 7571 (2000).

${ }^{8}$ N. W. A. van Uden, H. Hubel, D. A. Faux, A. C. Tanczos, B. Howlin, and D. J. Dunstan, J. Phys.: Condens. Matter 15, 1577 (2003).

${ }^{9}$ P. J. Berryman, D. A. Faux, and D. J. Dunstan, Phys. Rev. B 76, 104303 (2007).

${ }^{10}$ H. Hubel, D. A. Faux, R. B. Jones, and D. J. Dunstan, J. Chem. Phys. 124, 204506 (2006).

${ }^{11}$ S. Dixit, W. C. K. Poon, and J. Crain, J. Phys.: Condens. Matter 12, L323 (2000).

${ }^{12}$ N. W. A. van Uden, H. Hubel, D. A. Faux, D. J. Dunstan, and C. A. Royer, High Press. Res. 23, 205 (2003).

${ }^{13}$ CRC Handbook of Chemistry and Physics, 87 th ed., edited by D. R. Lide (CRC, Boca Raton, FL, 2006).

${ }^{14}$ Y. Melendez-Pagan and D. Ben-Amotz, J. Phys. Chem. B 104, 7858 (2000).

${ }^{15}$ K. S. Schweizer and D. Chandler, J. Chem. Phys. 76, 2296 (1982).

${ }^{16}$ M. R. Zakin and D. R. Herschbach, J. Chem. Phys. 89, 2380 (1988).

${ }^{17}$ M. R. J. Dack, Chem. Soc. Rev. 4, 211 (1975).
${ }^{18}$ E. V. Ivanov and V. K. Abrosimov, J. Struct. Chem. 46, 856 (2005).

${ }^{19}$ J. H. Hilderbrand and R. L. Scott, Regular Solutions (PrenticeHall, Englewood Cliffs, NJ, 1962).

${ }^{20}$ A. F. Barton, J. Chem. Educ. 48, 156 (1971).

${ }^{21}$ A. F. M. Barton, Handbook of Solubility Parameters and Other Cohesion Parameters, 2nd ed. (CRC, Boca Raton, FL, 1991).

${ }^{22}$ M. Kalweit and D. Drikakis, Phys. Rev. B 74, 235415 (2006).

${ }^{23}$ S. Viscardy, J. Servantie, and P. Gaspard, J. Chem. Phys. 126, 184513 (2007).

${ }^{24}$ W. Smith and T. R. Forester, J. Mol. Graphics 14, 136 (1996).

${ }^{25}$ Y. Marcus, Introduction to Liquid State Chemistry (Wiley, New York, 1977).

${ }^{26}$ P. Atkins, Elements of Physical Chemistry, 4th ed. (Oxford University Press, New York, 2005).

${ }^{27}$ K. Johnson, J. A. Zollweg, and K. E. Gubbins, Mol. Phys. 78, 591 (1993).

${ }^{28}$ W. G. Hoover, W. T. Ashurst, and R. Grover, J. Chem. Phys. 57, 1259 (1972).

${ }^{29}$ R. D. Bach, P. Y. Ayala, and H. B. Schlegel, J. Am. Chem. Soc. 118, 12758 (1996).

${ }^{30}$ E. Demiralp, T. Çağin, and W. A. Goddard, Phys. Rev. Lett. 82, 1708 (1999).

${ }^{31}$ C. A. English and J. A. Venables, Proc. R. Soc. London, Ser. A 340, 57 (1974).

${ }^{32}$ A. Laaksonen, P. Kusalik, and I. M. Svishchev, J. Phys. Chem. 101, 5910 (1997).

${ }^{33}$ W. P. Krekelberg, V. Ganesan, and T. M. Truskett, J. Chem. Phys. 124, 214502 (2006).

${ }^{34}$ Y. Ishibashi, T. Mishina, and J. Nakahara, Phys. Status Solidi B 243, 1159 (2006).

${ }^{35}$ K. Kendall, Molecular Adhesion and Its Applications: The Sticky Universe (Kluwer, Dordrecht/Plenum, New York, 2001).

${ }^{36}$ M. Kodaka, J. Phys. Chem. B 105, 5592 (2001).

${ }^{37}$ M. Kodaka, J. Phys. Chem. B 108, 1160 (2004). 